\title{
SIRT3 deficiency exacerbates p53/Parkin-mediated mitophagy inhibition and promotes mitochondrial dysfunction: Implication for aged hearts
}

\author{
YAN LI ${ }^{1 *}$, YING MA $^{1,2^{*}}$, LIQIANG SONG ${ }^{3 *}$, LU YU $^{4}$, LE ZHANG $^{1}$, \\ YINGMEI ZHANG ${ }^{1}$, YUAN XING ${ }^{5}$, YUE YIN $^{5}$ and HENG MA ${ }^{5}$ \\ ${ }^{1}$ Department of Cardiology, Xijing Hospital, The Fourth Military Medical University, Xi'an, Shaanxi 710032; \\ ${ }^{2}$ Department of Cardiology, The Fourth People's Hospital of Shaanxi Province, Xi'an, Shaanxi 710043; \\ Departments of ${ }^{3}$ Respirology, ${ }^{4}$ Pathology and ${ }^{5}$ Physiology, The Fourth Military Medical University, \\ Xi'an, Shaanxi 710032, P.R. China
}

Received August 18,2017; Accepted February 8, 2018

DOI: $10.3892 /$ ijmm.2018.3555

\begin{abstract}
Mitochondrial dynamics have critical roles in aging, and their impairment represents a prominent risk factor for myocardial dysfunction. Mitochondrial deacetylase sirtuin (SIRT) 3 contributes greatly to the prevention of redox stress and cell aging. The present study explored the role of SIRT3 on myocardium aging. Western blot analysis demonstrated that SIRT3 expression levels were significantly lower in the myocardia of aged mice compared with young mice. Immunoprecipitation and western blot assays indicated that the activity of mitochondrial manganese superoxide dismutase (MnSOD) and peroxisome proliferator-activated receptor $\gamma$ coactivator (PGC)- $1 \alpha$ was reduced in the aged heart. To further explore the association between SIRT3 and myocardial senescence, SIRT3 heart-specific knockout (SIRT3 ${ }^{-1}$ ) mice were used in the present study. The results revealed that obvious features of aging were present in the myocardium of SIRT $^{-/}$mice, including mitochondrial protein dysfunction, enhanced oxidative stress, and energy metabolism dysfunction. SIRT3 deficiency impaired Parkin-mediated mitophagy by increasing p53-Parkin binding and blocking the mitochondrial translocation of Parkin in cardiomyocytes. Injection of
\end{abstract}

Correspondence to: Dr Yan Li, Department of Cardiology, Xijing Hospital, The Fourth Military Medical University, 17 Changle West Road, Xi'an, Shaanxi 710032, P.R. China

E-mail: profleeyan@163.com

Dr Heng Ma, Department of Physiology, School of Basic Medical Sciences, The Fourth Military Medical University, 17 Changle West Road, Xi'an, Shaanxi 710032, P.R. China

E-mail: hmhm1610@sina.com

*Contributed equally

Key words: cardiac aging, sirtuin 3, mitophagy, mitochondrial homeostasis, Parkin autophagy agonist CCCP significantly increased the mitochondrial Parkin level in young wild-type hearts but not in aged hearts; the effect was less pronounced in SIRT3 ${ }^{-/}$hearts. These data suggest that CCCP-induced Parkin translocation was reduced in aged and SIRT ${ }^{-/}$hearts. CCCP-induced mitochondrial clearance, which could be rescued by autophagy antagonist bafilomycin-A1, was markedly weakened in aged and SIRT3 $3^{-/}$hearts vs. young hearts. SIRT3 deficiency exacerbated p53/Parkin-mediated mitophagy inhibition and disrupted mitochondrial homeostasis, suggesting that loss of SIRT3 may increase the susceptibility of aged hearts to cardiac dysfunction. Therapeutic activation of SIRT3 and improved mitochondrial function may ameliorate the symptoms of cardiac aging.

\section{Introduction}

Aging is among the most prominent risk factors for human diseases, including Alzheimer's disease, cataract, glaucoma, Parkinson's disease, arteriosclerosis, and heart failure (1). At present, the biological basis of aging is largely unknown, although several hypotheses have been proposed, such as the genetic theory of aging, radical theory, and decreased immune function theory (2). A growing body of evidence supports a critical role of mitochondrial homeostasis in the aging process (3-7). Damage and dysfunction in mitochondria are important factors in a range of human disorders because these organelles serve an irreplaceable role in energy production and cell metabolism $(6,8-10)$. Mitochondria are highly dynamic, and the regulation of mitochondrial quality control is subtle. Mitochondrial homeostasis is tightly modulated by two pathways: Biogenesis and mitochondrial autophagy, termed mitophagy (11). Mitophagy is a selective degradation process of mitochondria that are damaged or stressed $(12,13)$. Several studies have reported that mitophagy occurs in senescent myocardium and may have an important role in cardioprotection $(14,15)$.

Sirtuins is a protein family of nicotinamide adenine dinucleotide (NAD)-dependent deacetylases, which serve important roles in regulating cell stress, metabolism, growth, 
aging and apoptosis (16). Recently, several members of the family have been reported to be associated with autophagy for their deacetylation function (17). Sirtuin 3 (SIRT3) is the only family member that is highly expressed in the population of longevity (18). As a typical mitochondrial sirtuin family member, SIRT3 counteracts oxidative stress, defends against cell apoptosis, and prevents cell aging and tumorigenesis (19-22). Some studies have reported that SIRT3 reduces the level of reactive oxygen species (ROS) in the myocardium by deacetylating the transcription factor forkhead box (Fox)O3a, thus increasing the expression of its target genes, manganese superoxide dismutase (MnSOD) and catalase (CAT) $(23,24)$. Other reports have also demonstrated that SIRT3 increases the activity of these antioxidant enzymes through nuclear factor $\mathrm{\kappa B}$ and protects tissue from ROS-induced injury (25). SIRT3 levels in the murine heart can be increased by hypertrophic agonists in response to pressure overload and exercise. Furthermore, a recent study demonstrated that mouse mitochondrial dysfunction caused by loss of SIRT3 strongly contributed to obesity-related heart failure (26). However, the role of SIRT3 in regulating mitochondrial homeostasis in the myocardium remains largely unknown.

The present study aimed to explore the role of SIRT3 on mitochondrial homeostasis in the aged myocardium. The results demonstrated that SIRT3 knockout (KO) greatly inhibited p53/Parkin-mediated mitophagy. This inhibition disrupted mitochondrial homeostasis and resulted in irreversible mitochondrial dysfunction.

\section{Materials and methods}

Animals and ethics statements. Heart-specific SIRT3 KO $\left(\mathrm{SIRT3}^{--}\right)$weaned mice $(\mathrm{n}=54)$ and wild-type (WT) C57BL mice $(n=96)$ were purchased from the Jackson Laboratory (Ben Harbor, ME, USA) in the present study. The age of the male WT or KO mice used in the present study were as follows: Young (4 months), aged (20 months), and aged + aerobic intermittent training (AIT) (20 month old mice that underwent 13 weeks of AIT). To determine the effect of SIRT3 on Parkin-mediated cardiac mitophagy, specific mitophagy agonist CCCP (5 mg $\mathrm{CCCP} / \mathrm{kg}$ body weight, dissolved in DMSO; cat no. C2759; Sigma-Aldrich), alone or with the autophagy antagonist bafilomycin-A1 (12 $\mu \mathrm{g} / \mathrm{kg}$ body weight, dissolved in DMSO, cat. no. ab120497; Abcam, Cambridge, UK), was injected intraperitoneally into the WT young, WT aged, and SIRT3 KO mice $12 \mathrm{~h}$ ( $\mathrm{n}=8$ for each group) prior to subsequent detections. As a control, an equivalent dose of DMSO was injected into the littermates of the CCCP injection group. The mice were housed in a humidity- and temperature-controlled institutional laboratory animal facility where they had access to food and water ad libitum under a 12-h light/dark cycle. For sampling, $1 \%$ pentobarbital sodium was used to anesthetize the mice $(50 \mathrm{mg} / \mathrm{kg})$. The mice were euthanized immediately following the operation in a Mobile Anesthetic Workstation (Harvard Apparatus; Harvard Bioscience Inc., Cambridge, MA). All animal handling and experimental procedures described in the present study were approved by the Institutional Animal Care and Use Committee of the Fourth Military Medical University (Xi'an, China), and in compliance with the Guidelines for the Care and Use of Laboratory Animals (27).
Aerobic intermittent training (AIT). AIT was performed on aged or SIRT3 KO mice to evaluate the impacts of aerobic excise on SIRT3 activity and myocardial mitochondrial functions. The procedure was performed as follows: 1 week adaptive treadmill training at a speed of $15 \mathrm{~m} / \mathrm{min}$ (30 $\mathrm{min} /$ day, 5 days/week), followed by 12 weeks formal intermittent aerobic exercise at regularly changing speed $(1 \mathrm{~h} /$ day, 5 days/week). For each formal exercise section, the mouse first did warm-up exercise (40-50\% of maximum oxygen uptake) for $10 \mathrm{~min}$, followed by seven cycles of $4 \mathrm{~min}$ high-intensity (80-85\% VO2 max, speed of $23 \mathrm{~m} / \mathrm{min}$ ) and $3 \mathrm{~min}$ low-intensity intermittent aerobic exercise $(65-75 \% \mathrm{VO} 2 \mathrm{max}$, speed of $12 \mathrm{~m} / \mathrm{min}$ ). At the end of each section, the mouse had a $1 \mathrm{~min}$ cool-down period.

Mitochondria/cytosol fractionation. Mitochondria and cytosol protein compartments were fractionated with Mitochondria/Cytosol Fractionation Kit (BioVision, Inc., Milpitas, CA, USA) according to the manufacturer's instructions.

Co-Immunoprecipitation assay (co-IP). Myocardial samples were collected from each group $(\mathrm{n}=8)$, separately homogenized in Nonidet P-40 (NP-40) IP lysis buffer $(50 \mathrm{mM}$ Tris, $0.1 \%$ NP-40, $150 \mathrm{mM} \mathrm{NaCl}$ and $2 \mathrm{mM}$ EDTA; $\mathrm{pH} 7.5$; Beyotime Institute of Biotechnology, Shanghai, China) containing protease inhibitor (Invitrogen; Thermo Fisher Scientific, Inc., Waltham, MA, USA), and centrifuged at $12,000 \mathrm{x} \mathrm{g}$ for $10 \mathrm{~min}$ at $4^{\circ} \mathrm{C}$. Supernatants were collected, incubated with the primary antibody overnight at $4{ }^{\circ} \mathrm{C}$, and then mixed with protein G-Sepharose Fast Flow beads (Merck KGaA, Darmstadt, Germany) that were pre-equilibrated in lysis buffer. The primary antibodies used for IP were as follows: MnSOD (1:200; cat. no. S5069) and PGC-1 $\alpha$ (1:100; cat. no. AV40129) obtained from Sigma-Aldrich (St. Louis, MO, USA); p53 (1:100; cat. no. ab26) and Parkin (1:100; cat. no. ab15954) were obtained from Abcam (Cambridge, UK). The beads were collected by centrifugation $(3,000 \mathrm{x} \mathrm{g}$ for $5 \mathrm{~min}$ ), washed and resuspended in an equal volume of 5xSDS loading buffer. Immunoprecipitated proteins were separated by $10 \%$ SDS-PAGE, and immunoblotting was performed as described below.

Western blotting. Myocardial proteins were extracted using radioimmunopreciptation assay buffer $(50 \mathrm{mM}$ Tris- $\mathrm{HCl}$, pH7.5, 2 mM EDTA, $0.5 \%$ deoxycholate, $150 \mathrm{mM} \mathrm{NaCl}, 1 \%$ Triton X-100, 0.1\% SDS, $1 \mathrm{mM} \mathrm{Na3VO4}$ and $1 \mathrm{mM}$ PMSF). A total of $60 \mathrm{mg}$ of proteins from each sample was separated by $12 \%$ SDS-PAGE and transferred to polyvinylidene difluoride membranes at $120 \mathrm{~V}$ for $2 \mathrm{~h}$. The membranes were briefly washed with methanol and left to dry for 15 min to enhance the protein binding. The membranes were blocked with $5 \%$ bovine serum albumin (BSA; Invitrogen; Thermo Fisher Scientific, Inc.) in TBS-T and then incubated with primary antibodies overnight at $4^{\circ} \mathrm{C}$. For loading control, the membranes were probed with anti-GAPDH or tubulin (for non-mitochondrial proteins; 1:600; cat. no. sc-25778 and sc-69971, respectively, Santa Cruz Biotechnology, Inc., Dallas, TX, USA) or anti-cytocchrome $c$ oxidase complex (COX) IV (for mitochondrial proteins; 1:600; cat. no. HPA002485; Sigma-Aldrich) antibodies in TBS-T for 
$1 \mathrm{~h}$. The membranes were next incubated with horseradish peroxidase-conjugated goat anti-rabbit secondary antibody (1:2,000 dilution) in TBS-T for $1 \mathrm{~h}$. Finally, the membranes were incubated with enhanced chemiluminescence solution (Boehringer Mannheim; Roche Diagnostics GmbH, Mannheim, Germany), exposed in a ChemiDoc XRS imaging system, and analyzed with the Quantity One software (Bio-Rad Laboratories, Inc., Hercules, CA, USA). Rabbit/mouse monoclonal or polyclonal primary antibodies purchased from Sigma-Aldrich were used at dilutions: SIRT3 (1:500; cat. no. S4072), MnSOD (1:300), PGC-1 $\alpha$ (1:300), cyclin-dependent kinase inhibitor $2 \mathrm{~A}$ (p16; 1:800; cat. no. SAB4500072), acetyl-p53 (K317; 1:200; cat. no. SAB4503014), SIRT1 (1:500; cat. no. AV32386, SIRT1 served as a control to demonstrate the specificity of SIRT3 KO), glucose-regulated protein (GRP) 75 (1:400; cat. no. SAB4501454), NADH:Ubiquinone oxidoreductase subunit A9 (NDUFA9; 1:400; cat. no. WH0004704M1), and anti-acetyl lysine (1:200; cat. no. SAB5200090). In addition, rabbit polyclonal primary antibody against sequestosome 1 (p62; 1:400; cat. no. 23214) was obtained from Cell Signaling Technology, Inc. (Danvers, MA, USA). Antibodies against general control of amino acid synthesis 5-like 1 (GCN5L1; 1:500; cat. no. ab18381), p53 (1:800) and Parkin (1:400) were obtained from Abcam.

Measurement of protein content by UV spectrophotometry. A standard BSA solution at a concentration of $1 \mathrm{mg} / \mathrm{ml}$ was prepared, and aliquots of $0,1,2,3,4$ and $5 \mathrm{ml}$ of the standard solution were placed in six tubes, respectively. Each tube was filled with distilled water up to $5 \mathrm{ml}$. The first one was set as a blank control, and the absorption values of the standard solutions were successively measured under $280 \mathrm{~nm}$ UV light. The concentration of each tube was plotted to create a standard curve, which was then used to determine protein content of samples based on their A280 values.

Detection of enzyme activity and measurement of substrate content. Mice were euthanized by cervical dislocation. The hearts were rapidly removed, and a piece of full-thickness left ventricular myocardium was immediately clipped from each heart. The samples were snap-frozen in liquid nitrogen, and $0.3 \mathrm{~g}$ of each sample was placed in an ice-cold glass homogenizer. MnSOD and citrate synthase activity were detected with Manganese Superoxide Dismutase Activity Assay kit (Cayman Chemical Company, Ann Arbor, MI, USA) and Citrate Synthase Activity Assay kit (cat. no. ab119692; Abcam), respectively. ROS and lipofuscin were measured by Cellular Reactive Oxygen Species Detection Assay kit (cat. no. ab113851; Abcam) and Mouse Lipofuscin ELISA kit (Sigma-Aldrich). ATP and malondialdehyde (MDA) were detected with ATP Determination kit (Invitrogen; Thermo Fisher Scientific, Inc.) and OxiSelect TBARS Assay kit (Cell Biolabs, Inc., San Diego, CA, USA), respectively. Each detection or measurement was performed according to the manufacturer's instructions. The aforementioned measurements were performed in 12 mice per group to minimize the variation between individual animals and to reduce the false-positive/positive effect of the detection methods.

$\beta$-galactosidase ${ }^{+}$cell staining and counting. Myocardium $(0.1 \mathrm{~g})$ was sampled from the WT young, WT aged, and
SIRT3 KO mice. The tissue samples were shredded and then digested with $200 \mathrm{U} / \mathrm{ml}$ collagenase I for $\sim 2 \mathrm{~h}$ to obtain dissociated cardiomyocytes. The cardiomyocytes were stained with Senescence $\beta$-Galactosidase Staining kit (Cell Signaling Technology, Inc.) according to the manufacturer's instructions and analyzed with a flow cytometer (BD Biosciences, Franklin Lakes, NJ, USA).

Transmission electron microscopy. For transmission electron microscopy examination, the full-thickness left ventricular myocardium was mechanically dissected. The tissues were fixed in $3 \%$ glutaraldehyde in $0.1 \mathrm{M}$ cacodylate buffer ( $\mathrm{pH} 7.4$ ) at $4^{\circ} \mathrm{C}$ for $24 \mathrm{~h}$, post-fixed in $1 \% \mathrm{OsO}_{4}$ in the same buffer for $1 \mathrm{~h}$, dehydrated in graded ethanols, and embedded in Epon 812. Thin (60-90 nm) sections were used for ultrastructural evaluation using a $100 \mathrm{SX}$ transmission electron microscope (JEOL Inc., Peabody, MA, USA) operating at $80 \mathrm{kV}$.

Statistical analysis. Data are expressed as mean \pm standard error of the mean. One-way analysis of variance followed by the LSD test was used for multiple comparisons. All statistical tests were performed using SPSS v. 19.0 (IBM Corp., Armonk NY, USA). $\mathrm{P}<0.05$ was considered to indicate a statistically significant difference.

\section{Results}

SIRT3-mediated mitochondrial protein deacetylation is attenuated by aging but partially mitigated by AIT. The expression levels of SIRT3 protein in aged mouse myocardium were significantly lower compared with young mouse myocardium (Fig. 1A and B). Consistent with the change in SIRT3 protein levels, the overall acetylation of mitochondrial proteins was markedly elevated in the senile myocardium (Fig. 1C). However, the levels of the conserved mitochondrial acetyltransferase GCN5L1 did not differ significantly among the young, the aged and AIT groups $(\mathrm{F}=0.175, \mathrm{P}=0.844$; Fig. 1A and B). These results suggested that increase of overall acetylation in senile myocardium might be associated with downregulation of SIRT3; therefore, elevation of SIRT3 level might enhance the deacetylation of mitochondrial proteins in senile myocardium.

Previous studies have demonstrated that AIT is beneficial for the expression of mammalian SIRT3 in adult myocardium (28). In the present study, the effects of AIT on myocardial SIRT3 expression and acetylation of mitochondrial proteins in aged mice were investigated. The results demonstrated that myocardial SIRT3 expression could be partially rescued by AIT (Fig. 1A and B). Likewise, the deacetylation of mitochondrial proteins was also partially increased by AIT (Fig. 1C).

Oxidative stress and energy metabolism dysfunction are increased in aged myocardium. Mitochondrial MnSOD is important in mitigating oxidative stress (29), which is one of the main manifestations of aging. Mitochondrial proteins were isolated from young and aged WT myocardia, and the expression and activity of MnSOD were detected. Western blot and fluorescence spectrophotometric analyses revealed that the levels of MnSOD in aged myocardium were significantly lower compared with young myocardium $(\mathrm{F}=13.261$, 


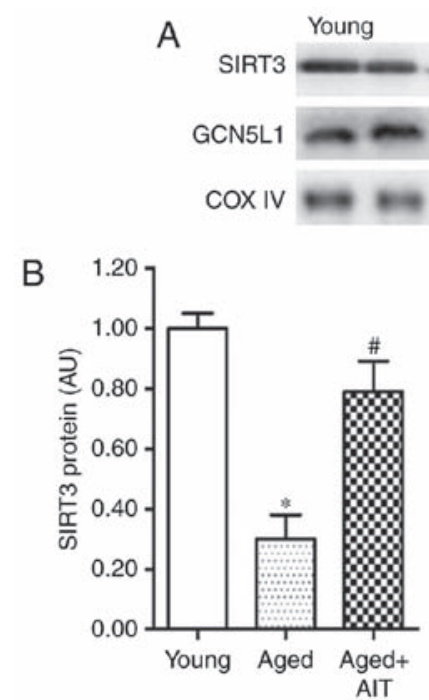

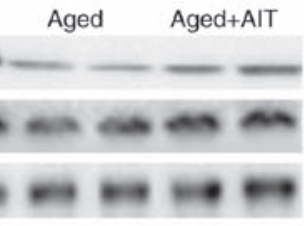

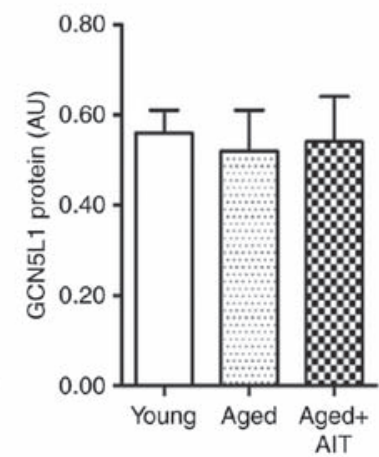

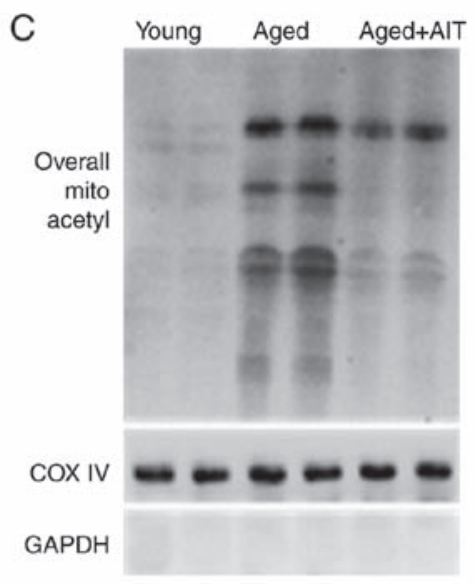

Figure 1. SIRT3 and SIRT3-mediated mitochondrial protein deacetylation are reduced in the aged myocardium. (A) Representative blots and (B) quantification of western blot analysis for the levels of SIRT3 and acetyltransferase GCN5L1 in the myocardial mitochondria of young, aged, and aged+AIT mice. Myocardial mitochondrial samples were separately isolated from WT mice aged as follows: Young (4 months), aged (20 months), and aged+AIT (20 month old mice that underwent 13 weeks of AIT). (C) The overall acetylation levels of mitochondrial protein in the myocardia of young, aged and aged+AIT mice. $n=8$ per group. "P<0.05 compared with the young; ${ }^{*} \mathrm{P}<0.05$ compared with both the young and the aged. SIRT3, sirtuin 3; GCN5L1, general control of amino acid synthesis 5-like 1; AIT, aerobic intermittent training; WT, wild-type; COX IV, cytochrome $c$ oxidase complex IV.

A

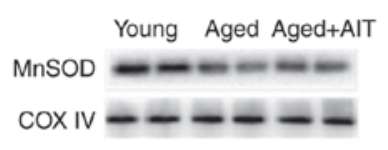

$\mathrm{D}$

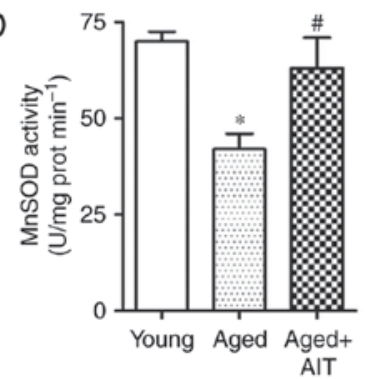

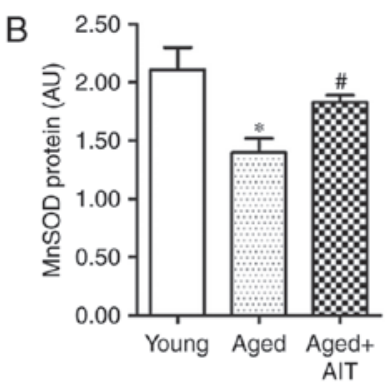

C IP:
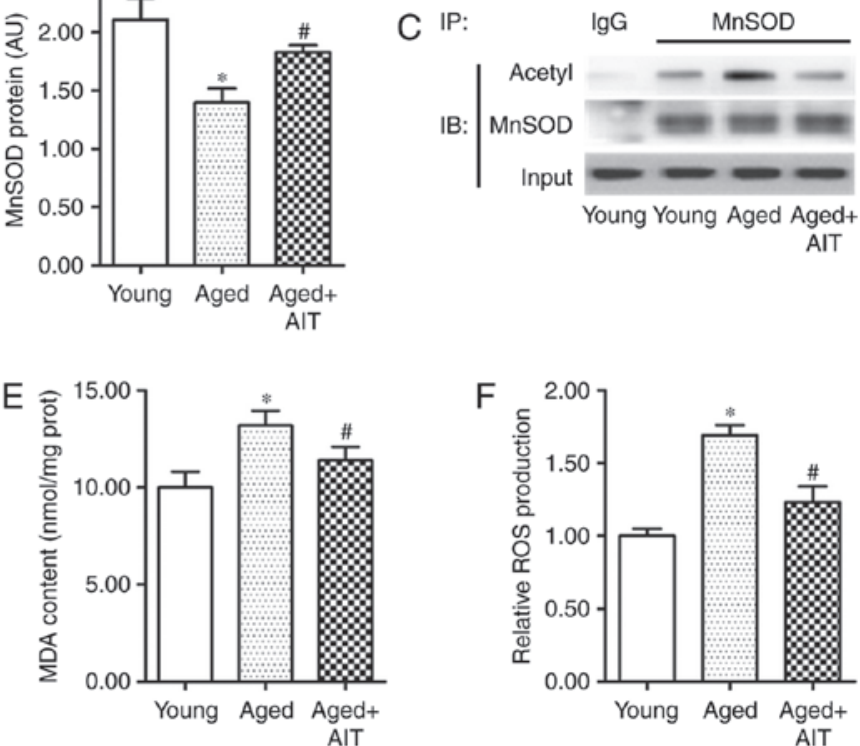

$\mathrm{F}$

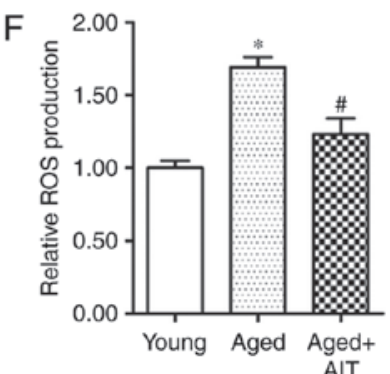

Figure 2. Expression and activity of MnSOD are reduced in the aged myocardium. Expression of MnSOD in the myocardial mitochondria of young, aged, and aged+AIT groups were detected with (A) western blot analysis and (B) UV spectrophotometry. (C) The acetylation levels of mitochondrial MnSOD were assayed by IP analysis. (D) Activity of MnSOD in the young, aged, and aged+AIT myocardia was analyzed with a MnSOD activity assay kit. (E) MDA content and (F) ROS production were detected with a MDA Quantitation Assay kit and a Cellular Detection Assay kit, respectively. $\mathrm{n}=8$ per group for (A-C), and $\mathrm{n}=12$ per group for $(\mathrm{D}-\mathrm{F}) .{ }^{*} \mathrm{P}<0.05$ compared with the young; ${ }^{*} \mathrm{P}<0.05$ compared with both the young and the aged. MnSOD, manganese superoxide dismutase; AIT, aerobic intermittent training; IP, immunoprecipitation; MDA, malondialdehyde; ROS, reactive oxygen species; COX IV, cytochrome $c$ oxidase complex IV; IB, immunoblotting.

$\mathrm{P}<0.001$; Fig. 2A and B). Co-IP analysis demonstrated that the acetylation of MnSOD in the aged myocardium was markedly elevated (Fig. 2C). In addition, the activity of MnSOD in aged myocardium was $\sim 60 \%$ of that in the young myocardium (Fig. 2D). In the aged+AIT group, the expression levels, activity and deacetylation of MnSOD were less affected (Fig. 2A-D). As key factors in oxidative stress, MDA and ROS were upregulated by 30 and $70 \%$, respectively, in the aged myocardium compared with the young (Fig. 2E and F). This upregulation was partially eliminated by AIT (Fig. 2E and F).

Energy metabolism dysfunction is also a main manifestation of aging. PGC-1 $\alpha$ is a key regulator of energy metabolism (30), and its expression and activity are commonly measured as a method to evaluate the energy metabolism of young and aged myocardia. PGC-1 $\alpha$ had lower expression, deacetylation and activity in aged myocardium compared with young (Fig. 3A-D). 
A
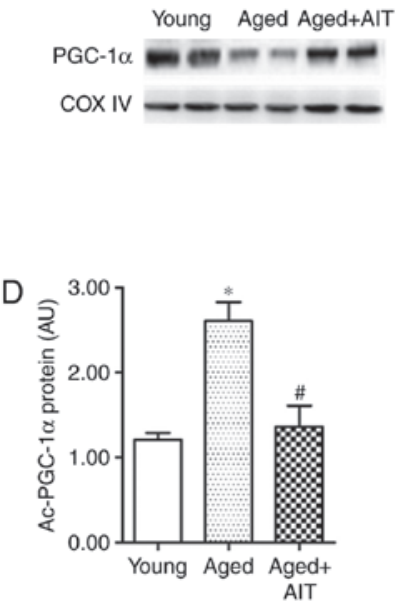

B
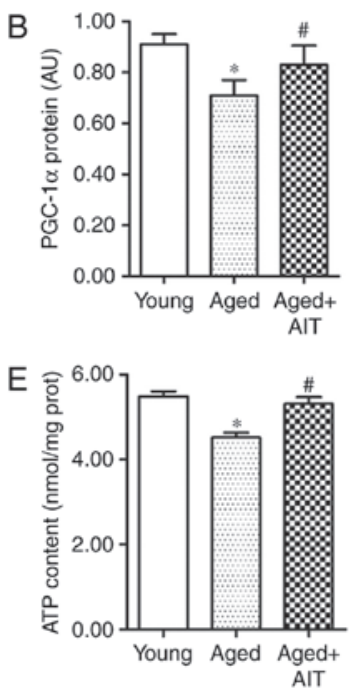

C
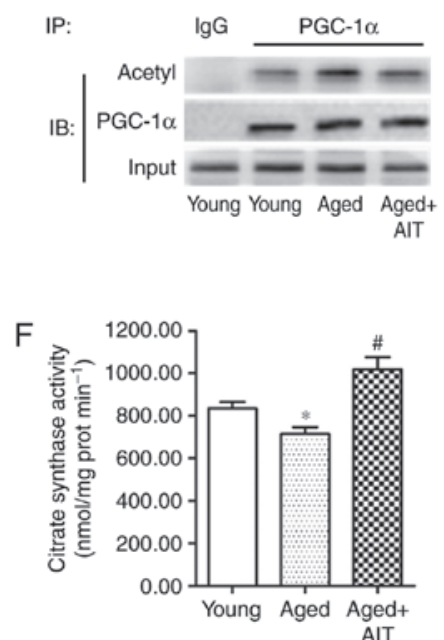

Figure 3. Expression and activity of PGC-1 $\alpha$ are reduced in the aged myocardium. Expression of PGC-1 $\alpha$ in the myocardial mitochondria of young, aged and aged+AIT groups were detected with (A) western blot analysis and (B) UV spectrophotometry. The acetylation levels of mitochondrial PGC-1 $\alpha$ were examined using (C) IP analysis and (D) mass spectrometry. (E) ATP content and (F) citrate synthase activity were assayed with ATP Determination kit and Citrate Synthase Activity Assay kit, respectively. $\mathrm{n}=8$ per group. ${ }^{*} \mathrm{P}<0.05$ compared with the young; ${ }^{*} \mathrm{P}<0.05$ compared with both the young and the aged. $\mathrm{PGC}-1 \alpha$, peroxisome proliferator-activated receptor $\gamma$ coactivator-1 $\alpha$; AIT, aerobic intermittent training; IP, immunoprecipitation; COX IV, cytochrome $c$ oxidase complex IV; IB, immunoblotting; Ac, acetylated.

A
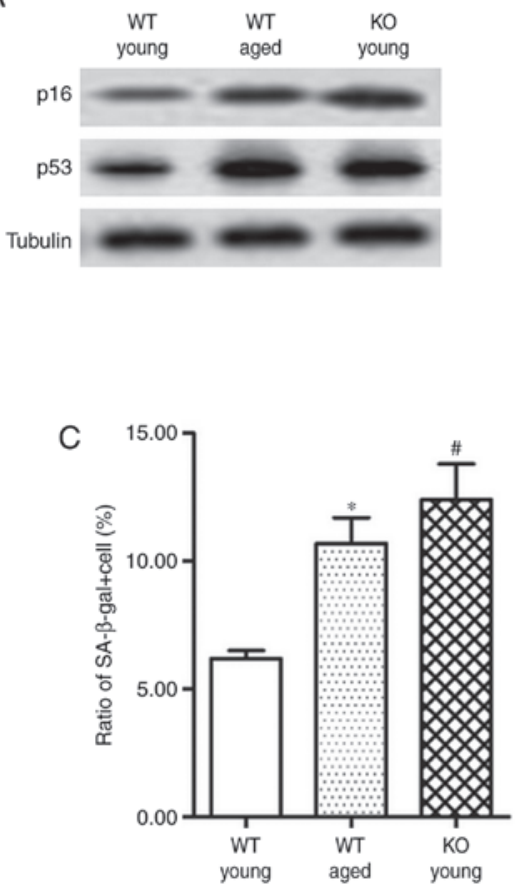
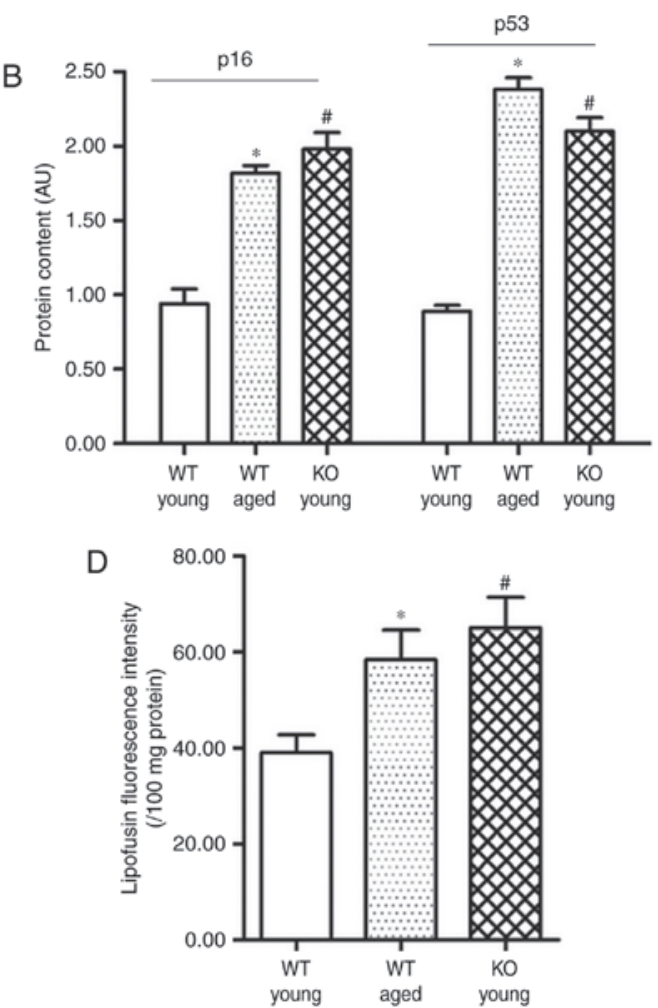

Figure 4. Levels of the senescence markers p16 and p53 are upregulated in SIRT3 ${ }^{-\digamma}$ mice. Detection of p16 and p53 protein levels in the WT young, WT aged, and SIRT3 KO myocardia by (A) western blotting and (B) UV spectrophotometry. (C) The proportion of senescence associated (SA)- $\beta$-galactosidase ${ }^{+}$cells in the WT aged and SIRT3 KO myocardia were significantly elevated compared with the WT young group. The cardiomyocytes obtained from WT young, WT aged and SIRT3 KO mice were stained with senescence $\beta$-Galactosidase Staining kit and analyzed by flow cytometry. (D) Lipofuscin content in WT aged and SIRT3 KO myocardia was significantly more abundant compared with the WT young group. $\mathrm{n}=8$ per group. ${ }^{*} \mathrm{P}<0.05$ compared with the young; ${ }^{*} \mathrm{P}<0.05$ compared with both the young and the aged. p16, cyclin-dependent kinase inhibitor 2A; p53, tumor protein p53; SIRT3, sirtuin 3; WT, wild-type; KO, knockout.

In addition, the ATP content and citrate synthase activity were demonstrated to be reduced in aged myocardium compared with the young (Fig. 3E and F), suggesting a decrease in the metabolic ability of aged myocardium. Similar to the changes of MnSOD, the reduced PGC- $1 \alpha$ expression, deacetylation, and activity, as well as the energy metabolism, were improved by AIT (Fig. 3). These results and the expression profiles of SIRT3 in aged myocardium suggested that SIRT3 reduction 
A
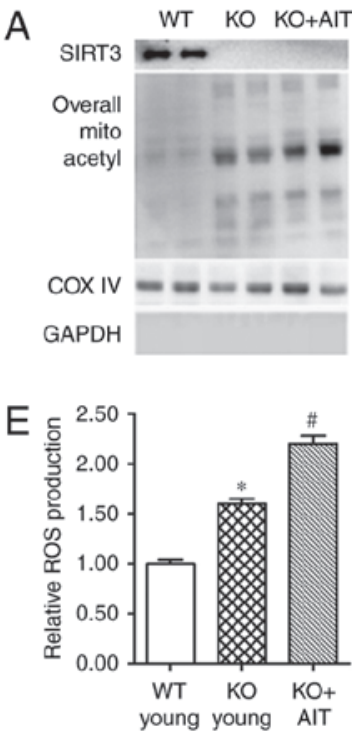
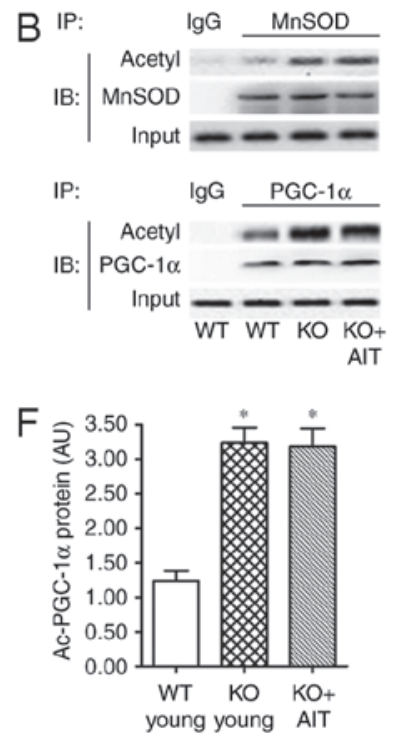
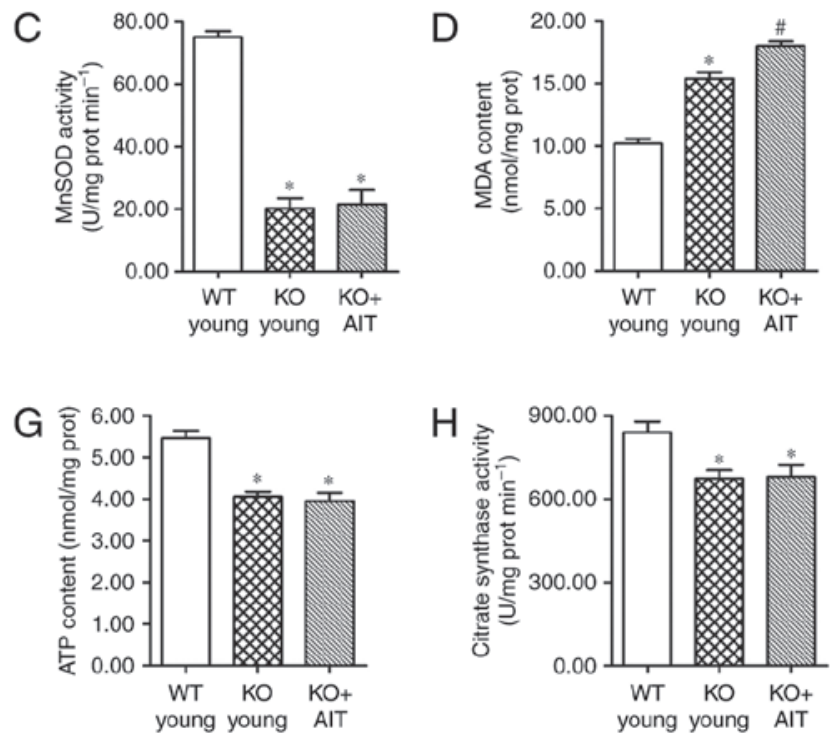

Figure 5. The myocardium of SIRT3 ${ }^{-/-}$mice displays features of enhanced oxidative stress and energy metabolism dysfunction. (A) Detection of SIRT3 and mitochondrial protein acetylation levels in the WT young, SIRT3 ${ }^{-/}(\mathrm{KO})$, and KO+AIT myocardia by western blotting. (B) Detection of acetyl/total MnSOD and acetyl/total PGC-1 $\alpha$ by IP/western blot analyses. (C) MnSOD activity, (D) MDA content and (E) ROS production in the myocardia of WT young, KO young and $\mathrm{KO}+\mathrm{AIT}$ mice. $(\mathrm{F})$ Acetylation levels of PGC-1 $\alpha,(\mathrm{G})$ ATP content and $(\mathrm{H})$ citrate synthase activity in the myocardia of WT young, KO young, and KO+AIT mice. $\mathrm{n}=8$ per group. ${ }^{*} \mathrm{P}<0.05$ compared with the WT group; ${ }^{*} \mathrm{P}<0.05$ compared with both the WT and the KO groups. SIRT3, sirtuin 3 ; WT, wild-type; KO, knockout; AIT, aerobic intermittent training; MnSOD, manganese superoxide dismutase; PGC-1 $\alpha$, peroxisome proliferator-activated receptor $\gamma$ coactivator-1 $\alpha$; IP, immunoprecipitation; MDA, malondialdehyde; ROS, reactive oxygen species; COX IV, cytochrome $c$ oxidase complex IV; IB, immunoblotting; Ac, acetylated.

was closely related to acetylation and downregulation of MnSOD and PGC-1 $\alpha$-induced aging in mitochondrial homeostasis.

Myocardium of SIRT3 ${ }^{--}$mice displays obvious features of aging, including mitochondrial protein dysfunction, enhanced oxidative stress and energy metabolism dysfunction. To explore the association between SIRT3 and myocardial senescence, SIRT3 KO (SIRT3 ${ }^{-/-}$) mice were used in subsequent experiments. Compared with the young WT mice (aged 4 months), the levels of senescence marker genes p16 and p53 in the adult SIRT ${ }^{-/-}$mice were upregulated by $\sim 80$ and $140 \%$, respectively (Fig. 4A and B). In addition, both the proportion of $\beta$-galactosidase ${ }^{+}$cells and the lipofuscin content in the SIRT $^{-/-}$mice were increased by $\sim 50 \%$ (Fig. 4C and D).

As in the analyses for senescent myocardium, the overall acetylation of mitochondrial proteins and the expression and acetylation of MnSOD and PGC-1 $\alpha$ were then analyzed in the SIRT3 ${ }^{-1-}$ myocardium. Western blot analysis revealed that the overall acetylation was robustly enhanced in the absence of SIRT3 (Fig. 5A). A strong increase in the acetylation of MnSOD and PGC-1 $\alpha$ accompanied by a sharp decrease in their activity was also detected (Fig. 5B, C and F). Consistent with the alteration of the MnSOD and PGC-1 $\alpha$ levels, production of MDA and ROS was greatly elevated, while ATP content was reduced and citrate synthase activity was inhibited (Fig. 5D, E, G and H). However, in the absence of SIRT3, AIT had no effect on the deacetylation of mitochondrial proteins or energy metabolism (Fig. 5A, B and F-H) and it even exacerbated oxidative stress in myocardial mitochondria (Fig. 5D and E). These data indicated that myocardial mitochondrial protein damage and dysfunction caused by SIRT3 deletion could not be mitigated by AIT.
SIRT3 deficiency impairs Parkin-mediated mitophagy by increasing p53-Parkin binding. Mitophagy serves important roles in mitochondrial self-renewal and inhibition of mitochondrial dysfunction during aging or post-injury. It was speculated that SIRT3 deficiency may have caused abnormal mitophagy, therefore, further experiments explored autophagosome morphology and signal transduction in mitophagy regulation. Electron microscopy revealed that autophagic vacuoles were reduced in aged and SIRT3 ${ }^{-/}$myocardium compared with adult WT myocardium (Fig. 6A).

Several previous studies have suggested that p53 is likely to be involved in aging and myocardial mitophagy $(31,32)$. Therefore, in the present study western blot analysis was used to detect the impact of aged SIRT3 and SIRT3 deficiency on the level and activity of p53. The results demonstrated that aged SIRT3 raised the acetylation level of p53, and SIRT3 deficiency further increased the acetylation level (Fig. 6B). The co-IP analysis revealed that in aged myocardium the interaction between SIRT3 and p53 was reduced, and in $\mathrm{SIRT3}^{-/}$myocardium the interaction was hardly detectable (Fig. 6C). These results indicated that deacetylation of p53 was regulated by SIRT3 and the deacetylating function on p53 was attenuated by aging or SIRT3 deficiency.

Parkin is a key factor in mitophagy, and its translocation into mitochondria is essential for inducing mitophagy $(33,34)$. Parkin can be bound by activated p53 (acetylated), which then blocks its translocation (35). The co-IP analysis for p53 and SIRT3 revealed that $\mathrm{p} 53$-Parkin binding increased in aged myocardium and even further elevated in the SIRT3 ${ }^{-/}$myocardium (Fig. 6D and E). These results indicated that p53 was activated and bound to Parkin, which was likely an important reason for impaired Parkin-mediated mitophagy in the aged and SIRT ${ }^{-/-}$myocardia. 
A
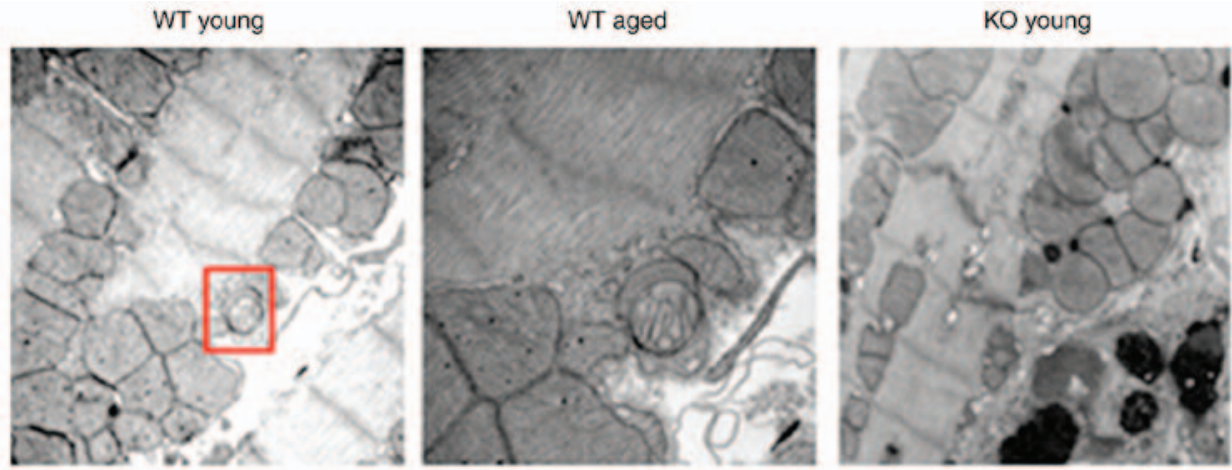

B
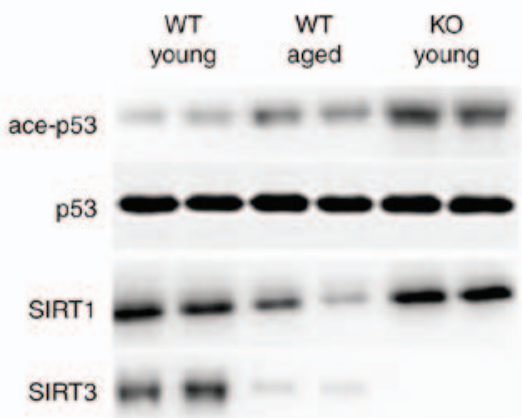

D IP:

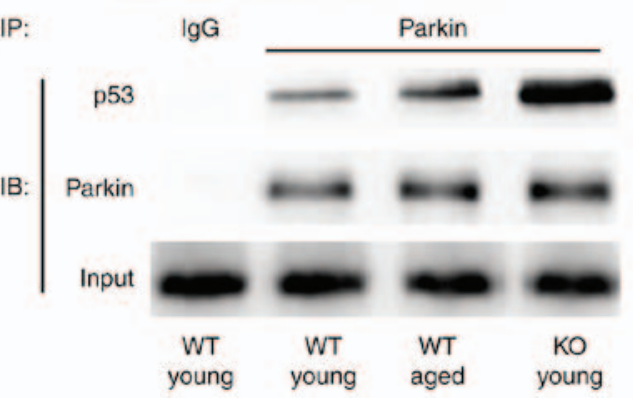

C IP:

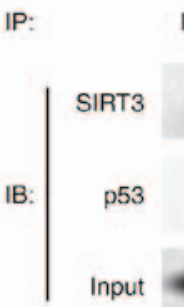

E IP

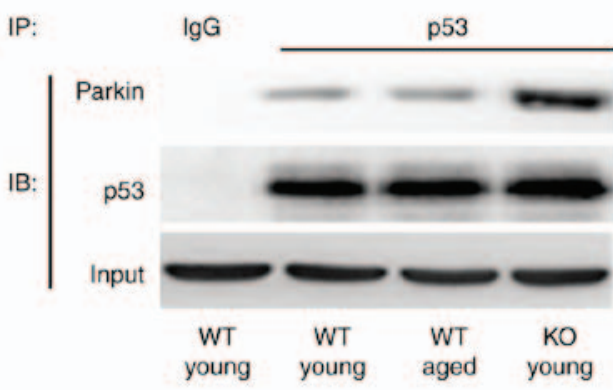

Figure 6. The myocardium of SIRT3 ${ }^{-/}$mice displays features of enhanced oxidative stress and energy metabolism dysfunction. (A) Representative images from transmission electron microscopy analysis for autophagic vacuoles in the WT young, WT aged, and SIRT ${ }^{1 /-}$ (KO) myocardia. Original magnification, $\mathrm{x} 8,000$. The red box demonstrated a representative autophagic vacuole. (B) Detection of acetyl/total p53 by western blot analysis. SIRT1 herein served as a control to demonstrate the specificity of SIRT3 KO. (C) The interaction between p53 and SIRT3 in WT young, WT aged, and KO young myocardial mitochondria was determined by co-IP assay. p53 primary antibody was incubated with the mitochondrial proteins for IP; IgG was the negative control. Then a SIRT3 or p53 secondary antibody was applied in the following IB experiment. (D and E) The interaction between p53 and Parkin in WT young, WT aged and KO young myocardial mitochondria was determined by co-IP assay. n=8 per group. SIRT3, sirtuin 3; WT, wild-type; KO, knockout; p53, tumor protein p53; IP, immunoprecipitation; Ig, immunoglobulin; IB, immunoblotting; ace, acetylated.

SIRT3 is required to maintain Parkin-mediated mitophagy. To further validate that SIRT3 deficiency was involved in 553 re-acetylation, binding to Parkin and inhibition of Parkin-mediated mitophagy, a specific mitophagy agonist CCCP was intraperitoneally injected into young, aged and $\mathrm{SIRT}^{-/-}$mice (5 mg CCCP $/ \mathrm{kg}$ body weight). Twelve hours following injection, changes in the levels of the myocardial mitophagy markers Parkin and its translocation blocker p62 were detected. Western blot analyses demonstrated that the levels of Parkin and p62 did not differ among young, aged, and SIRT3 ${ }^{-1-}$ mice in cytosol or mitochondria compared with controls (Fig. 7A). CCCP administration resulted in a significant increase in the mitochondrial levels of Parkin and p62 in the young because it induced their translocation into mitochondria. Notably, the translocation was quite marked in the young mice, but not so obvious in the aged mice and even less in SIRT3 ${ }^{-/}$mice (Fig. 7A). These data suggested that CCCP-induced Parkin/p62 translocation was reduced in aged and SIRT3 ${ }^{-1-}$ hearts.
Finally, autophagy antagonist bafilomycin-A1 (Baf-A1), CCCP, or both were applied to young, aged, and SIRT3 $3^{-1-}$ mice. In the young cardiomyocytes, levels of mitochondrial chaperone GRP75 and mitochondrial ubiquinone NDUFA9, typical markers of mitochondrial clearance, were significantly downregulated following CCCP administration, but could be rescued by Baf-A1 administration (Fig. 7B). CCCP-induced mitochondrial clearance was markedly attenuated in aged and SIRT3 ${ }^{-1}$ hearts compared with the young (Fig. 7B). Thus mitochondria were impaired by blockage of autophagy in aged and SIRT $3^{-/}$hearts, and SIRT3 decline or deficiency was responsible for impaired Parkin-mediated mitophagy in senescent or SIRT3 $^{-1}$ hearts.

\section{Discussion}

Cardiac aging is characterized by hypertrophy and fibrosis of the heart, which results in increased susceptibility to stress, such as ischemia and hemodynamic overload. It has been 

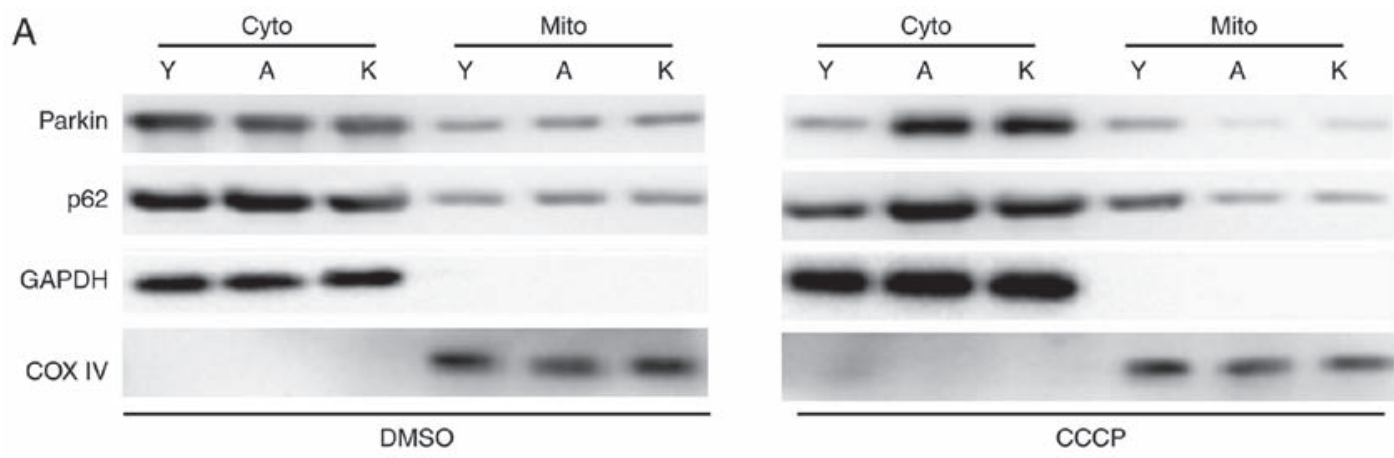

B

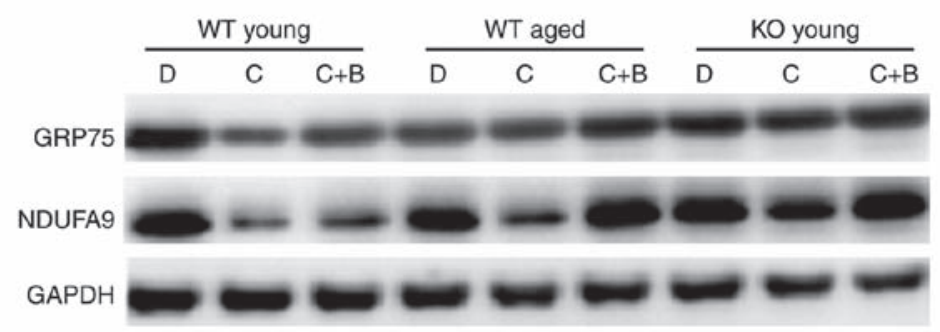

Figure 7. SIRT3 is required to maintain Parkin translocation and Parkin-mediated mitophagy. (A) Parkin mitochondrial translocation was blocked in WT aged and SIRT3 $3^{-1}$ (KO) myocardia when mitophagy was stimulated by the agonist CCCP. CCCP was injected (5 mg/kg body weight) intraperitoneally into WT young, WT aged, and KO mice $(\mathrm{n}=5)$. As a control, an equivalent dose of DMSO was injected into the littermates of the CCCP injection group (n=4). Following $12 \mathrm{~h}$, the heart tissues were sampled, and the levels of Parkin and of its translocation blocker p62 were detected by western blot analysis. (B) CCCP-induced mitochondrial clearance was markedly weakened in WT aged and KO myocardia. CCCP, alone or with the autophagy antagonist bafilomycin-A1, was injected intraperitoneally into the WT young, WT aged, and KO mice ( $\mathrm{n}=8$ per group). As a control, an equivalent dose of DMSO was injected into the littermates of the CCCP injection group $(\mathrm{n}=8)$. Following $12 \mathrm{~h}$, the heart tissues were sampled, and the levels of mitochondrial chaperone GRP75 and mitochondrial ubiquinone NDUFA9 were detected by western blot analysis. $\mathrm{n}=8$ per group. SIRT3, sirtuin 3; WT, wild-type; KO, knockout; DMSO, dimethyl sulfoxide; p62, sequestosome 1; GRP75, glucose-regulated protein 75; NDUFA9, NADH dehydrogenase 1 alpha subcomplex 9; COX IV, cytochrome $c$ oxidase complex IV; cyto, cytoplasmic; mito, mitochondrial; Y, WT young group; A, WT aged group; K, SIRT3 knockout group; D, DMSO-treated group; C, CCCP-treated group; $\mathrm{C}+\mathrm{B}, \mathrm{CCCP}+$ bafilomycin-A1-treated group.

demonstrated that the incidence of heart failure dramatically increases with aging (36). However, the exact causes of heart aging remain unknown. The heart is an organ in which mitochondria are enriched in high density in order to meet the high energy-demand and to maintain redox stress homeostasis (37). Therefore, mitochondrial dysfunction caused by oxidative stress is believed to be an important cause of cardiac aging and failure. Several lines of evidence have revealed that SIRT3 is involved in cardiac aging related to oxidative stress and mitochondrial dysfunction. However, the role and mechanisms of SIRT3 in modulating heart aging and mitochondrial function require further study. In the present study, SIRT3 deficiency was demonstrated to be associated with cardiac aging due to, at least in part, its inhibition of mitophagy and its effect on mitochondrial functions related to oxidative stress and energy metabolism.

SIRT3, an aging-related deacetylase, has been previously reported to protect cardiomyocytes from stress-mediated cell death and improve mitochondrial respiration $(38,39)$. In fact, its roles in many important biological processes are mostly based on its deacetylating function. In the present study, the role of SIRT3 in aged myocardium was investigated. Acetylation of p53 and other mitochondrial proteins was demonstrated to be upregulated in aged and SIRT ${ }^{-/}$myocardia. Strikingly, Kim et al (40) also demonstrated that acetyl-lysine was present in $>20 \%$ of mitochondrial proteins, including many oxidative stress-related proteins, longevity regulators and metabolism enzymes. SIRT3 ${ }^{-/}$mice are well-suited for experiments to investigate the impact of SIRT3 deficiency on the biological characteristics of the myocardium. Several reports have revealed that SIRT3 knockdown/knockout exacerbates ischemia-reperfusion injury, myocardium failure, and nutrientor exercise-induced stress in the heart $(20,41,42)$. In the present study, it was demonstrated that protein deacetylation disorder, enhanced oxidative stress and energy metabolism dysfunction, which occurred in aged hearts, were also present in SIRT3hearts. These data indicated that SIRT3 deficiency likely had a strong impact on the regulation of myocardial mitochondrial function under the aging condition.

In the present study, SIRT3 deficiency was confirmed to be associated with the elevated oxidative stress and disturbed energy metabolism, which were recognized as typical features of cardiac aging. Mitochondria are involved in a range of other processes besides energy supply, such as signaling, cellular ion homeostasis, oxidative stress, apoptotic and necrotic cell death. In the present study, the impacts of SIRT3 on oxidative stress and energy metabolism in mitochondria were explored. Mitochondrial MnSOD is very important in resisting mitochondrial ROS-induced oxidative stress (43), which is an important part of radical theory. In aged myocardial mitochondria, the level and activity of MnSOD were sharply reduced by SIRT3. The mechanism for SIRT3 influencing ROS-induced oxidative stress was previously demonstrated to involve SIRT3 deacetylating FoxO3a and then upregulating its target genes, $M n S O D$ and CAT $(23,24)$. Moderate intensity AIT is often recommended for weight loss and prevention of chronic disorders, including aging-related diseases (44-46). In the present study, it was demonstrated that MnSOD suppression could be 
attenuated by aging but partially maintained by AIT. Similar results were obtained for PGC-1 $\alpha$, the key regulator of energy metabolism. Hence, protein deacetylation disorder, enhanced oxidative stress and energy metabolism dysfunction caused by aging could be alleviated, but not eliminated, by physical training. Additionally, an important role of SIRT3 in these processes may be speculated.

Furthermore, SIRT3 deficiency suppressed p53/Parkinmediated mitophagy, in turn leading to the inhibition of damaged mitochondrial clearance. Mitophagy is vital to maintaining mitochondrial homeostasis, and its inhibition can lead to mitochondrial dysfunction and abnormal cell functions. Parkin, an E3 ligase originally discovered as mutated in monogenic forms of Parkinson's disease, was recently found to translocate specifically to uncoupled mitochondria and to induce autophagy of damaged mitochondria (47). Present in the cytosol, Parkin can be targeted by activated cytosolic p53, which disrupts its translocation to damaged mitochondria and subsequent mitophagy (35). In the present study, Parkin-mediated mitophagy was significantly inhibited in aged or SIRT3 deficient animals. It is likely that, compared with normal conditions, SIRT3 in aged or SIRT3 ${ }^{-1-}$ myocardium cannot maintain p53 deacetylation, leading to higher amounts of activated p53, more binding with Parkin, and consequent inhibition of Parkin translocation. The results from the co-IP analysis demonstrated that the interaction between SIRT3 and p53 was reduced in aged or SIRT3 ${ }^{-1-}$ myocardium, which supported the aforementioned hypothesis.

Overall, SIRT3 deficiency raised the acetylation levels of mitochondrial proteins and disrupted mitochondrial homeostasis. On one hand, proteins such as MnSOD and PGC-1 $\alpha$ are important to resist the redox stress and maintain normal mitochondrial biogenesis; increased acetylation suppressed MnSOD and PGC- $1 \alpha$ activity and exacerbated mitochondrial dysfunction in aged hearts. On the other hand, with a lack of SIRT3, p53-Parkin binding was enhanced, the translocation of Parkin was blocked, and Parkin-mediated mitophagy was inhibited; mitochondria could not renew through mitophagy. Of note, the functions of mitochondria in a range of biological processes, as well as a comprehensive understanding of the role of SIRT3 in regulating mitochondria dynamics, will require further studies in the future.

In conclusion, decreased SIRT3 could disrupt mitochondrial homeostasis and increase the susceptibility of the aged heart to cardiac injury. The present results suggest that therapeutic activation of SIRT3 and improved mitochondrial function may ameliorate the symptoms of cardiac aging.

\section{Acknowledgements}

Not applicable.

\section{Funding}

This study was supported by research grants from the National Natural Science Foundation of China (81570252; 81500195; 81170184) and Military Foundation (CWS14J065). Availability of data and materials. The datasets used and/or analyzed during the present study are available from the corresponding author on reasonable request.

\section{Availability of data and materials}

The analyzed data sets generated during the study are available from the corresponding author upon reasonable request.

\section{Authors' contributions}

YL and LQS and HM conceived and designed the study. YL and YM drafted the manuscript. YM and LY conducted the experiments. LZ, YX and YY analyzed and interpreted the data; LQS and YMZ critical revised the manuscript for important intellectual content. YL and HM final approval of the drafted manuscript. All authors have read and approved the final manuscript.

\section{Ethics approval and consent to participate}

All animal handling and experimental procedures described in the present study were approved by the Institutional Animal Care and Use Committee of the Fourth Military Medical University (Xi'an, China), and in compliance with the Guidelines for the Care and Use of Laboratory Animals (27).

\section{Consent for publication}

Not applicable.

\section{Competing interests}

The authors declare that they have no competing interests.

\section{References}

1. Niccoli T and Partridge L: Ageing as a risk factor for disease. Current Biol 22: R741-R752, 2012.

2. Rattan SI: Rationale and methods of discovering hormetins as drugs for healthy ageing. Expert Opin Drug Discov 7: 439-448, 2012.

3. Schmitt K, Grimm A, Kazmierczak A, Strosznajder JB, Götz J and Eckert A: Insights into mitochondrial dysfunction: Aging, amyloid- $\beta$, and tau-A deleterious trio. Antioxid Redox Signal 16: 1456-1466, 2012.

4. Biala AK, Dhingra R and Kirshenbaum LA: Mitochondrial dynamics: Orchestrating the journey to advanced age. J Mol Cell Cardiol 83: 37-43, 2015.

5. Nasrallah CM and Horvath TL: Mitochondrial dynamics in the central regulation of metabolism. Nat Rev Endocrinol 10: 650-658, 2014.

6. Bullon P, Newman HN and Battino M: Obesity, diabetes mellitus, atherosclerosis and chronic periodontitis: A shared pathology via oxidative stress and mitochondrial dysfunction? Periodontol 2000 64: 139-153, 2014

7. Schiavi A and Ventura N: The interplay between mitochondria and autophagy and its role in the aging process. Expe Gerontol 56: $147-153,2014$.

8. Currais A: Ageing and inflammation-A central role for mitochondria in brain health and disease. Ageing Res Rev 21: 30-42, 2015.

9. Sorrentino G, Comel A, Mantovani F and Del Sal G: Regulation of mitochondrial apoptosis by Pin1 in cancer and neurodegeneration. Mitochondrion 19: 88-96, 2014.

10. Paradies G, Paradies V, Ruggiero FM and Petrosillo G: Cardiolipin and mitochondrial function in health and disease. Antioxid Redox Signal 20: 1925-1953, 2014.

11. Palikaras K and Tavernarakis N: Mitochondrial homeostasis: The interplay between mitophagy and mitochondrial biogenesis. Exp Gerontol 56: 182-188, 2014.

12. Chu CT, Bayır H and Kagan VE: LC3 binds externalized cardiolipin on injured mitochondria to signal mitophagy in neurons: Implications for Parkinson disease. Autophagy 10: 376-378, 2014. 
13. Youle RJ and Narendra DP: Mechanisms of mitophagy. Nat Rev Mol Cell Biol 12: 9-14, 2011.

14. Thomas RL and Gustafsson AB: Mitochondrial autophagy: An essential quality control mechanism for myocardial homeostasis. Circ J 77: 2449-2454, 2013.

15. Moyzis AG, Sadoshima J and Gustafsson AB: Mending a broken heart: The role of mitophagy in cardioprotection. Am J Physiol Heart and Circ Physiol 308: H183-H192, 2015.

16. Houtkooper RH, Pirinen E and Auwerx J: Sirtuins as regulators of metabolism and healthspan. Nat Rev Mol Cell Biol 13: 225-238, 2012.

17. Lee IH, Yun J and Finkel T: The emerging links between sirtuins and autophagy. Methods Mol Biol 1077: 259-271, 2013.

18. Zhang B, Cui S, Bai X, Zhuo L, Sun X, Hong Q, Fu B, Wang J, Chen X and Cai G: SIRT3 overexpression antagonizes high glucose accelerated cellular senescence in human diploid fibroblasts via the SIRT3-FOXO1 signaling pathway. Age (Dordr) 35: 2237-2253, 2013.

19. Ahn BH, Kim HS, Song S, Lee IH, Liu J, Vassilopoulos A, Deng CX and Finkel T: A role for the mitochondrial deacetylase Sirt3 in regulating energy homeostasis. Proc Nat Acad Sci USA 105: 14447-14452, 2008.

20. Jing E, Emanuelli B, Hirschey MD, Boucher J, Lee KY, Lombard D, Verdin EM and Kahn CR: Sirtuin-3 (Sirt3) regulates skeletal muscle metabolism and insulin signaling via altered mitochondrial oxidation and reactive oxygen species production. Proc Nat Acad Sci 108: 14608-14613, 2011.

21. Haigis MC, Deng CX, Finley LW, Kim HS and Gius D: SIRT3 is a mitochondrial tumor suppressor: A scientific tale that connects aberrant cellular ROS, the Warburg effect, and carcinogenesis. Cancer Res 72: 2468-2472, 2012.

22. Alhazzazi TY, Kamarajan P, Verdin E and Kapila YL: SIRT3 and cancer: Tumor promoter or suppressor? Biochim Biophys Acta 1816: 80-88, 2011.

23. Aldakkak M, Stowe DF, Chen Q, Lesnefsky EJ and Camara AK: Inhibited mitochondrial respiration by amobarbital during cardiac ischaemia improves redox state and reduces matrix $\mathrm{Ca}^{2+}$ overload and ROS release. Cardiovasc Res 77: 406-415, 2008.

24. Sundaresan NR, Gupta M, Kim G, Rajamohan SB, Isbatan A and Gupta MP: Sirt3 blocks the cardiac hypertrophic response by augmenting Foxo3a-dependent antioxidant defense mechanisms in mice. J Clin Invest 119: 2758-2771, 2009.

25. Chen CJ, Fu YC, Yu W and Wang W: SIRT3 protects cardiomyocytes from oxidative stress-mediated cell death by activating NF- $\kappa$ B. Biochem Biophys Res Commun 430: 798-803, 2013.

26. Sack MN: Emerging characterization of the role of SIRT3mediated mitochondrial protein deacetylation in the heart. Am J Physiol-Heart Circ Physiol 301: H2191-H2197, 2011.

27. Garber JC, Barbee RW, Bielitzki JT, Clayton L, Donovan J, Hendriksen C, Kohn DF, Lipman NS, Locke PA, Melcher J, et al: Guide for the care and use of laboratory animals. Washington DC, The National Academic Press, pp220, 2011.

28. Jiang HK, Miao Y, Wang YH, Zhao M, Feng ZH, Yu XJ, Liu JK and Zang WJ: Aerobic interval training protects against myocardial infarction-induced oxidative injury by enhancing antioxidase system and mitochondrial biosynthesis. Clin Exp Pharmacol Physiol 41: 192-201, 2014.

29. Merksamer PI, Liu Y, He W, Hirschey MD, Chen D and Verdin E: The sirtuins, oxidative stress and aging: An emerging link. Aging (Albany NY) 5: 144-150, 2013.

30. Adamovich Y, Shlomai A, Tsvetkov P, Umansky KB, Reuven N, Estall JL, Spiegelman BM and Shaul Y: The protein level of PGC-1 $\alpha$, a key metabolic regulator, is controlled by NADH-NQO1. Mol Cell Biol 33: 2603-2613, 2013.
31. Rufini A, Tucci P, Celardo I and Melino G: Senescence and aging: The critical roles of p53. Oncogene 32: 5129-5143, 2013.

32. Hoshino A, Matoba S, Iwai-Kanai E, Nakamura H, Kimata M, Nakaoka M, Katamura M, Okawa Y, Ariyoshi M, Mita Y, et al: p53-TIGAR axis attenuates mitophagy to exacerbate cardiac damage after ischemia. J Mole Cell Cardiol 52: 175-184, 2012.

33. Saito T and Sadoshima J: Molecular mechanisms of mitochondrial Autophagy/mitophagy in the heart. Circ Res 116: 1477-1490, 2015.

34. Herman AM and Moussa CE: The ubiquitin ligase parkin modulates the execution of autophagy. Autophagy 7: 919-921, 2011.

35. Hoshino A, Mita Y, Okawa Y, Ariyoshi M, Iwai-Kanai E, Ueyama T, Ikeda K, Ogata T and Matoba S: Cytosolic p53 inhibits Parkin-mediated mitophagy and promotes mitochondrial dysfunction in the mouse heart. Nat Commun 4: 2308, 2013.

36. Sadoshima J: Sirt3 targets $\mathrm{mPTP}$ and prevents aging in the heart. Aging 3: 12-13, 2011.

37. Sack MN: The role of SIRT3 in mitochondrial homeostasis and cardiac adaptation to hypertrophy and aging. J Mol Cell Cardiol 52: 520-525, 2012.

38. Samant S, Pillai V, Wolfgeher D and Gupta M: SIRT3 protects cardiomyocytes from Doxorubicin-induced mitochondrial damage and Cell-death by Activating Opa1. Circulation 130 (Suppl 2): A14664, 2014.

39. Cheung KG, Cole LK, Xiang B, Chen K, Ma X Myal Y, Hatch GM, Tong Q and Dolinsky VW: Sirtuin-3 (SIRT3) protein attenuates Doxorubicin-induced Oxidative Stress and improves mitochondrial respiration in $\mathrm{H} 9 \mathrm{c} 2$ cardiomyocytes. J Biol Chem 290: 10981-10993, 2015.

40. Kim SC, Sprung R, Chen Y, Xu Y, Ball H, Pei J, Cheng T, Kho Y, Xiao H, Xiao L, et al: Substrate and functional diversity of lysine acetylation revealed by a proteomics survey. Mol Cell 23: 607-618, 2006.

41. Sol EM, Wagner SA, Weinert BT, Kumar A, Kim HS, Deng CX and Choudhary $\mathrm{C}$ : Proteomic investigations of lysine acetylation identify diverse substrates of mitochondrial deacetylase sirt3. PLoS One 7: e50545, 2012.

42. Kawamura Y, Uchijima Y, Horike N, Tonami K, Nishiyama K, Amano T, Asano T, Kurihara Y and Kurihara H: Sirt3 protects in vitro-fertilized mouse preimplantation embryos against oxidative stress-induced p53-mediated developmental arrest. J Clin Invest 120: 2817-2828, 2010.

43. James AM, Collins Y, Logan A and Murphy MP: Mitochondrial oxidative stress and the metabolic syndrome. Trend Endocrinol Metab 23: 429-434, 2012.

44. Hosseinzadeh S, Dabidi Roshan V and Pourasghar M: Effects of intermittent aerobic training on passive avoidance test (shuttle box) and stress markers in the dorsal hippocampus of wistar rats exposed to administration of homocysteine. Iran J Psychiatry Behav Sci 7: 37-44, 2013.

45. Cardoso AM, Bagatini MD, Roth MA, Martins CC, Rezer JF, Mello FF, Lopes LF, Morsch VM and Schetinger MR: Acute effects of resistance exercise and intermittent intense aerobic exercise on blood cell count and oxidative stress in trained middle-aged women. Braz J Med Biol Res 45: 1172-1182, 2012.

46. Tanaka M, Sugawara M, Ogasawara Y, Izumi T, Niki K and Kajiya F: Intermittent, moderate-intensity aerobic exercise for only eight weeks reduces arterial stiffness: Evaluation by measurement of stiffness parameter and pressure-strain elastic modulus by use of ultrasonic echo tracking. J Med Ultrason (2001) 40: 119-124, 2013

47. Narendra D, Tanaka A, Suen DF and Youle RJ: Parkin is recruited selectively to impaired mitochondria and promotes their autophagy. J Cell Biol 183: 795-803, 2008. 